\title{
La nouvelle Loi handicap
}

\author{
Béatrice IDIARD CHAMOIS
}

Institut Mutualiste Montsouris, Paris

\section{RÉSUMÉ}

Si la personne en situation de handicap à travers les siècles a été considérée tour à tour soit comme une image de maléfice, de pitié, de pêché, aujourd'hui quelle image et quels droits, la loi du 11/02/2005 sur l'égalité des droits et des chances, la participation et la citoyenneté des personnes handicapées lui apporte-t-elle de plus ? N'y-a-t- il pas encore un énorme fossé entre ce qui est écrit et ce qui est réellement ? Car depuis le XIX siècle (création de la première loi), des dizaines d'autres sont venues se rajouter. De même que le vocabulaire a changé au fil des époques, passant de mutilé, invalide à celui de handicapé.

Les différences énormes entre les chiffres officiels et ceux issue d'une enquête HID (Handicap Indépendance Incapacité), réalisée entre 1998 et 2001, reflète encore mieux le fait que l'on a encore du mal à bien définir les personnes en situation de handicap ; la population française vieillissant, les technologies s'améliorant, d'ici quelques années nous risquons d'être débordés par un taux important de personnes en situation de handicap, et nous en ferons partie un jour ou l'autre. La nouvelle loi du 11/02/2005 répond-elle à cette situation d'urgence ? A-t-elle cernée tous les problèmes en matière d'accessibilité, d'aides humaines, de scolarité de droits des personnes en situation de handicap ? N'est-elle pas une énième loi sur les personnes en situation de handicap qui ravie le législateur plus que les personnes concernées?

La nouvelle loi va s'axer sur quatre points. Tout d'abord le droit à compensation en créant la prestation compensatoire du handicap (PCH) qui remplace l'allocation compensatrice tierce personne (ACTP) et l'allocation frais professionnels (AFP) et l'allocation adulte handicapé (AAH), qui existait déjà, le droit à la scolarité à tous les enfants en situation de handicap, le droit à l'emploi. Et enfin la création des maisons départementales du handicap (MDPH) qui regroupe différents acteurs impliqués actuellement dans l'accompagnement des personnes handicapées (conseil général, DDASS, CPAM, CAF, etc...).

Pour ce qui est du droit à compensation : création de la prestation de compensation du handicap ( $\mathrm{PCH})$, elle est basée maintenant sur cinq grands types d'aide, des aides humaines, techniques, d'aménagements du logement et du véhicule ainsi que des surcoûts de transport, des aides animalières, et enfin des aides exceptionnelles et spécifiques.

Outre les aides directes liées à la personne en situation de handicap, la nouvelle loi parle aussi de la mise en accessibilité (loi et décrets existants déjà depuis 1991 et 1994) des transports, des lieux recevant du publics etc... Mais la loi donne aussi une autre définition de l'accessibilité en étendant la mise en accessibilité par rapport à toutes les situations de handicap (moteur, sensoriel, cognitif, mental, etc...). Cette mise en accessibilité a une date butoir d'achèvement : 3 ans pour les transports, 5 ans pour les universités et les préfectures, et 10 ans pour les autres bâtiments recevant du public.

Une autre innovation de cette loi est que la formation soit continue, soit initiale des professionnels de santé, du secteur médico-social aux personnes en situations de handicap (origine des handicaps, innovations thérapeutiques, pédagogiques, accueil et accompagnement des personnes handicapées, l'annonce du handicap, etc...).

Mais les retards pris au fil des siècles ont laissé et laisseront encore beaucoup de personnes en situation de handicap sur le bord de la route. Car si il y a une prise de conscience de la part des politiques, les erreurs, les retards, et encore les imperfections de cette nouvelle loi feront du handicap une tolérance et non une insertion pour les années à venir.

II faut que les personnes réfléchissent aujourd'hui autrement au handicap, et cela quelques personnes l'ont compris en créant depuis 2002 un réseau de la commission européenne "Design for All » ou "Conception pour Tous 》 où il s'agit de développer des systèmes utilisables pour les personnes dites valides mais aussi pour les personnes en situation de handicap.

\section{Correspondance :}

Béatrice IDIARD CHAMOIS, Sage-femme - Institut Mutualiste Montsouris, 42 Boulevard Jourdan, 75014 PARIS. 
De notre coté, au niveau de la parentalité, de la naissance, à notre échelle, nous essayons d'y réfléchir car si la loi a prévu " tout " sur les parents valides qui ont en charge un enfant en situation de handicap, le vide reste total quant à la parentalité des personnes en situation de handicap. Ainsi, un travail s'ébauche, grâce à l'ensemble de l'équipe du Département Mère-Enfant de l'Institut Mutualiste Montsouris, sur l'accueil de ces futurs parents avec l'aide débutante d'ESCAVIE (espace conseil pour l'autonomie en milieu ordinaire de vie) où ergothérapeute et assistant social participent à ce travail. Enfin la création d'une association parents handicapés, parents d'abord (APHAPA) au sein du Département Mère-Enfant.

Enfin, un dernier point qui ne concerne pas directement la nouvelle loi, mais dont je pense qu'il est important de parler. II s'agit de la loi de bioéthique d'août 2004 et diagnostic pré implantatoire, loi pour laquelle un débat s'est tenu dernièrement à l'Assemblée sur sa révision. Le vieux mythe de l'eugénisme refait surface, et malheureusement des hommes politiques de nos jours s'y adonnent avec l'aide des médias qui renvoient cette image de bébé parfait, de l'image du beau, du normal, du stéréotype. Et bien je n'en fait pas partie, et c'est tant mieux, je préfère de loin ma vie à beaucoup d'autres car même si l'on n'accepte jamais sa maladie ou son handicap, on vit avec, et quoiqu'on en pense, j'existe avec mes joies, mes peines, bref tout ce qui m'a permis de me construire, d'être devenue ce que je suis, peut-être une ingérable, une marginale, mais pour moi tout simplement un être humain, et aucune loi ne me changera.

Mots Clés : situation d'handicap, prestation compensatrice du handicap, formation, parentalité, accessibilité, loi de Bioéthique

\section{INTRODUCTION}

"Mettons-la en place pour que chacun trouve sa place ", ce slogan que vous avez du entendre durant l'année 2006 sur les ondes hertziennes découle de la nouvelle loi du 11 février 2005 pour l'égalité des droits et des chances, la participation et la citoyenneté des personnes handicapées. Elle tente de répondre à l'attente des personnes handicapées en matière de droit à compensation, de scolarité, d'emploi, d'accessibilité, et enfin à la création des maisons départementales des personnes handicapées (MDPH).

Mais aujourd'hui, qu'en est il vraiment de cette loi, les personnes en situation de handicap (expression que j'utiliserai par la suite) sont-elles vraiment au courant de ces changements, les personnels de santé (médecins, assistantes sociales, infirmières, etc...) connaissent-ils les différents articles de cette loi les concernant? N'est-ce pas encore, une fois de plus, une énième loi sur le handicap et qui petit à petit tombera dans l'oubli, mais ayant fait auparavant la part belle aux politiques ? Les personnes en situation de handicap vont-elles se retrouver insérées, et non plus tout simplement tolérées, dans une société qui sans cesse les rejette en leur dressant des obstacles de toute nature ?

Toutes ces interrogations, je me les suis posées et je vais tenter d'y répondre avec l'aide de mes recherches, en m'appuyant sur la loi, surtout sur ma propre expérience, le projet que nous avons mis en place à l'Institut Mutualiste Montsouris (IMM) dans le Département Mère-Enfant et le projet d'une association.

Mon ambition n'est pas de décortiquer la loi, mais de sensibiliser les personnels médicaux, para médicaux et sociaux aux situations de handicap, même si « ce n'est pas la peine car dans leur vie professionnelle il ne rencontreront peut être qu'un handicapé», réflexion faite par un médecin, car nous sommes tous condamnés à plus ou moins longue échéance, temporaire ou définitive, à être dans une situation de handicap.

C'est pourquoi je ferai un rappel sur le vocabulaire, la classification des situations de handicap, la place de la personne en situation de handicap suivant les époques, les chiffres, la définition selon la nouvelle loi, ainsi que la loi concernant les professionnels de santé, enfin j'aborderai la prise en charge dans notre Département Mère-Enfant, les maladies génétiques et la loi de Bioéthique 2004 , ainsi que l'avenir, puis je ferai un commentaire personnel sur cette loi.

\section{VOCABULAIRE}

Si le mot handicap est maintenant devenu un mot courant, ce n'est que dans les années 1950 que ce mot est apparu pour la première fois dans les lois françaises pour parler des travailleurs handicapés. Est considérée comme travailleur handicapé pour bénéficier des dispositions de la présente loi, toute personne dont les possibilités d'acquérir, ou de conserver un emploi sont effectivement réduites par suite d'une insuffisance ou d'une diminution de ses capacités physiques ou mentales. Ce mot vient de l'anglais (1827) hand in cap (la main dans le chapeau), qui était un jeu d'échange d'objets pratiqué en Grande Bretagne au XVIème siècle. Puis ce mot a été utilisé dans les milieux turfistes entre 1850 et 1872.

Auparavant on parlait plutôt d'invalide : qui n'est pas en état de mener une vie active, de travailler, du fait de sa mauvaise santé, de ses infirmitès, de ses blessures, etc...; d'infirme :1247, rare avant le XVIème siècle, du latin infirmus qui signifiait faible, au XVIIIème siècle, qui est atteint d'une ou plusieurs infirmités (spécialement d'infirmités incurables) ; d'estropié, de mutilé : personne qui a subi une mutilation, généralement par fait de guerre ou par accident, par exemple un amputé ; ou encore impotent : 1308, du latin impotens qui signifiait impuissant : qui, par une cause naturelle ou accidentelle, ne peut se mouvoir, ou ne se meut qu'avec une extrême difficulté, donc dépendant de l'aide d'autrui.

\section{CLASSIFICATION DES SITUATIONS DE HANDICAP}

On peut les classer selon les circonstances de leur acquisition:

- congénital ou acquis,

- maladie, accident ou vieillissement,

- évolutif ou non évolutif.

Ou bien selon le type :

- moteur,

- sensoriel,

- mental,

- physiques liés à une maladie chronique. 


\section{HISTOIRE}

Si l'on commence à entrevoir aujourd'hui un "embryon " d'insertion des personnes en situation de handicap, notre histoire nous fait comprendre aussi pourquoi autant de retard aujourd'hui. D'abord abandonnée, cachée, puis regroupée pour mieux être dirigée et contrôlée, expérimentée, exterminée, assistée, tout cela en accord avec la religion puis phagocytée dans un système de lois politiciennes, la personne en situation de handicap n'a jamais eu le choix de choisir sa vie, malgré la création de nombreuses associations depuis. Parfois par peur, indifférence, ou pitié de la part de ses semblables " sains ", elle se trouve toujours à devoir faire le premier pas en direction d'un monde dit « normal ».

Tout au long des siècles, les différentes situations de handicap ont connu des écarts considérables, par exemple l'éducation des sourds au droit à l'instruction et la reconnaissance de leur langue, ou bien celle des aveugles avec l'ancêtre du braille durant le siècle des lumières, tandis que le XIXème siècle, ère de l'industrialisation, voit le développement du matériel pour mutilés ou invalides (fauteuils, prothèses etc...).

\section{L'Antiquité}

Dans l'antiquité l'homme a très vite compris le besoin naturel d'entraide. En effet, la solidarité, l'entraide et le partage permettaient la survie d'un groupe d'hommes au sein d'un milieu hostile. Les premières traces d'entraide retrouvées ont été la prise en charge du rite funéraire par la communauté [18]. Paradoxalement, les nouveaux-nés difformes ou mutilés représentaient une menace pour ce même groupe d'hommes et étaient considérés comme un maléfice. II fallait s'en débarrasser au plus vite, c'était le signe de la colère des Dieux et la faute des hommes. Les bébés étaient alors abandonnés hors des villages dans la nature, loin du groupe social par décision du conseil des sages [21].

Pour Aristote les sourds n'avaient pas d'intelligence puisqu'ils n'avaient pas l'ouie, pour Platon logos voulait dire parole mais aussi intelligence, celui qui ne possédait pas la parole était idiot, les aveugles, débiles et sourds étaient épargnés [4].

Les Egyptiens, au contraire, n'hésitaient pas à représenter leurs dieux sous formes de personnages difformes, de nains [6].

Dans la culture hébraïque, l'infirmité apparaissait comme une impureté, il ne fallait présenter aucune tare pour pouvoir présenter les offrandes [21].

\section{Au moyen âge et jusqu'au XVIIème siècle}

Durant cette période, une partie de la société est sous le contrôle de l'église, qui dispose d'une grande richesse et d'une puissance considérable. Elle va considérer les infirmes, tout comme les pauvres, comme des personnes à qui l'on doit la charité, donc à secourir et non à exclure. Les riches donnent aux pauvres pour leur salut. Une exception pour les sourds qui avaient l'interdiction de participer aux rites religieux car considérés comme sans âme puisque sans parole. De même qu'ils n'avaient aucun droit civique (défense d'hériter, de se marier). lls se trouvaient exploités dans les campagnes et les villes car leur situation de handicap leur permettaient de " tout faire " et surtout de n'avoir aucun moyen de rébellion [4].

Les aveugles étaient considérés comme des personnages menteurs, antipathiques et faisaient l'objet de moquerie.
Les infirmes, s'ils n'étaient pas pris en charge par leur famille, étaient voués à la mendicité, ce sont les fameuses «Cours des miracles» où pauvres, gueux, infirmes, malades se côtoyaient.

Cependant, on voit apparaître par Saint Louis la première institution pour les aveugles vers 1260 toujours sous le contrôle de l'Eglise, les XV/XX. Les infirmes et les malades eux se retrouveront à l'Hôtel Dieu. Toujours pour s'assurer une main d'œuvre nombreuse et utile, les monarques vont créer pour certain corps de métiers, notamment les marins, des traités qui visent à les protéger en cas d'accident du travail ou d'invalidité. C'est le cas d'Aliénor d'Aquitaine en 1152, qui rédigea les Rôles d'Oléron, mesure reprise ensuite par Colbert en 1673 et développée. Henri IV étendra cette mesure aux mineurs en 1604 pour le remboursement des frais médicaux et chirurgicaux [18].

II fallait que la société puisse avoir à la fois une bonne image, celle de la charité mais aussi de contrôle et de mise à l'écart des infirmes.

Mais rapidement cette " image " va se détériorer, certainement à cause des nombreuses épidémies, famines et guerres. Les personnes infirmes vont être à nouveau l'image de l'impiété, du pêché et un produit du diable [6].

Cependant la médecine va s'intéresser aux monstruosités de façon scientifique, notamment Ambroise Paré ( " Des monstres et prodiges "1573), et créera aussi les premières prothèses des membres inférieurs et supérieurs.

Durant les XVIème et XVIIème siècles, on assistera à la période des bouffons, nombreux sont les monarques qui se faisaient représenter en peinture avec des nains.

La création des Invalides par Louis XIV, la création de l'Hôpital Général (1656) et l'interdiction de la mendicité en avril 1656 vont aboutir à l'enfermement total et définitif des infirmes, pauvres et fous. II faut que l'ordre social règne !

De plus en plus l'infirmité sera considérée comme marginale, la société ne mettant en œuvre aucune mesure éducative ou médicale en rapport avec les besoins des infirmes ; les gueux, criminels, infirmes, pauvres, malades, etc..., tous ceux que la société considère comme une menace à l'ordre social, le travailleur et l'inapte, le normal et le pathologique [21] seront enfermés dans des hospices, asiles ou orphelinats.

Pourtant, dans certains hospices, l'infirme, pour éviter l'oisiveté, devra fournir un travail (création d'une manufacture de chaussures, tapisseries, vêtements par Louvois à l'Hôtel des Invalides qui était d'abord réservé aux blessés de guerre).

\section{Le siècle des Lumières et la Révolution Française}

Une époque riche en bouillonnement d'idées, de créativité et de réflexion.

Parmi les philosophes, je citerai Montesquieu qui, dans « L'esprit des Lois " (1748), dit ceci : " L'Etat doit à tous les citoyens une subsistance assurée, la nourriture, un vêtement convenable et un genre de vie qui ne soit pas contraire à sa santé ".

De Diderot, dans sa " Lettre sur les aveugles à l'intention de ceux qui voient " (1749) qui sera suivie par " la lettre aux sourds-muets à l'intention de ceux qui entendent " $"$, je citerai cet extrait : " Les connaissances ont trois portes pour entrer dans notre âme, et nous en tenons une barricadée par le défaut de signes. Si l'on eut négligé les deux autres, nous en serions réduits à la condition 
des animaux. De même que nous n'avons que le serré pour nous faire entendre au sens du toucher, nous n'aurions que le cri pour parler à l'oreille. Madame, il faut manquer d'un sens pour connaître les avantages des symboles destinés à ceux qui restent ; et des gens qui auraient le malheur d'être sourds, aveugles et muets, ou qui viendraient à perdre ces trois sens par quelque accident, seraient bien charmés qu'il y ait une langue nette et précise pour le toucher ».

Puis la création de la première institution pour sourds muets et son système des « signes méthodiques " par l'Abbé de L'Epée (1712-1789) en 1776, repris à sa mort par l'Abbé Sicard. On fait émerger l'idée que les gestes pourraient exprimer la pensée humaine autant qu'une langue orale, et que même si l'Abbé de l'Epée n'a pas crée la langue des signes, (il a juste voulu transposer le français parlé en signes et cela a déstabilisé les sourds), il avait le mérite de les considérer comme des êtres humains. Avant lui, Jacob Rodrigue Pereire, précepteur dans les familles riches, parlait de démutisation des enfants sourdsmuets qu'ils avaient en charge, se refusant d'utiliser les gestes naturels des sourds, son apprentissage consistait à ce que l'élève pose sa main sur la gorge du professeur afin de sentir les vibrations et articulations.

Etienne de Fay, en 1710 surnommé aussi le « vieux sourd d'Amiens ", fut le premier professeur à enseigner en langage gestuel à des enfants. II avait réussi à devenir professeur et architecte à Amiens [4].

C'est en 1779 que le premier livre a été écrit par un sourd, Pierre Desloges : " Structure de la langue des signes ".

Jean Massieu, qui était l'élève de l'Abbé Sicard, deviendra le premier éducateur sourd à l'Institut de Paris, puis directeur de l'école de Rodez et de Lille. C'est à lui que l'on doit ce proverbe "La reconnaissance est la mémoire du cœur ".

A la Révolution Française les sourds bénéficient des droits de l'Homme en 1791.

Pour les aveugles c'est Valentin Haüy (1745-1822) qui créa I'Institution des enfants aveugles en 1786, école qui porte aujourd'hui le nom d'INJA (institut national des jeunes aveugles). D'abord tourné vers la surdité, ce sont deux évènements de sa vie qui vont l'orienter vers l'éducation des aveugles : tout d'abord en 1771 il assiste à une bien piètre représentation oủ des aveugles pensionnaires des $X V / X X$, affublés de déguisements ridicules et de lunettes opaques jouent de la musique discordante devant des assistants moqueurs, puis en mai 1784 il fit la connaissance d'un mendiant aveugle, François Lesueur, et, frappé de la finesse de son toucher capable de différencier des pièces de monnaie, il entreprit son instruction. Sous la Révolution, l'institution fut prise en charge par l'Etat, il du démissionner en 1802. II créa alors le Musée des aveugles (école privée pour aveugles étrangers), et partit en 1806 en Russie où il fonda une école qu'il dirigea pendant 11 ans $[19,20]$.

Mais malheureusement les autres infirmes restaient toujours enfermés dans des conditions terribles, surtout les malades mentaux, et vers la fin du XVIIIème siècle, Philippe Pinel invente la psychiatrie (1793) et on voit apparaître les premières cliniques orthopédiques et les premières remises au travail des infirmes physiques.

Toutes ces mesures découlent des cahiers de doléances et de la Déclaration des Droits de l'Homme et du Citoyen de 1793, article
21 : " Les secours sont une dette sacrée. La société doit la subsistance aux citoyens malheureux, soit en leur procurant un travail, soit en assurant les moyens d'exister à ceux qui sont hors d'état de travailler ". Ainsi l'Etat gère les hôpitaux et les maisons de charité, et met en place la gratuité de la médecine et de l'hospitalisation pour les pauvres, la pension pour les vieillards, les pères de famille nombreuse, les infirmes. Le comité de santé, présidé par La Rochefoucault Liancourt, pense que « L'assistance est un devoir de la société, il faut développer l'emploi pour diminuer l'indigence " [18]

Notons un bémol : La loi Le Chapelier de 1791 qui interdit toute coalition, corporation, association, et compagnonnage. Elle eut pour effet d'interdire les syndicats et le droit de grève, elle fut abrogée le 25/05/1864 par la loi Ollivier.

\section{Le XIXème siècle ou ère de l'industrialisation et de la première loi}

L'assistance religieuse et familiale va peu à peu faire place à un système obligatoire fondé sur la solidarité nationale mais encore organisé en un réseau disparate. En effet, l'industrialisation va faire apparaître une nouvelle classe sociale : les ouvriers. $\mathrm{Ce}$ qui va amener à une multitude de problèmes : la longueur des journées de travail, le manque de prévention, les nouvelles techniques mal maîtrisées vont multiplier les accidents du travail et la maladie. On va donc voir apparaître les premières sociétés mutualistes dès 1800 , la première caisse d'épargne est crée en 1818 , de même que les sociétés de secours mutuels, interdites par la loi Le Chapelier, continuent à opérer dans la clandestinité. De plus, des sociétés de bienfaisance se multiplient et les plus riches utilisent leur fortune pour faire le bien aux plus nécessiteux. L'état, quant à lui, intervient peu sauf dans des décrets ou lois sur le travail des enfants (décret de 1813 sur les enfants dans les mines, loi de 1841 sur la durée du temps de travail des enfants) [18]. Mais il faudra attendre 1893 pour que la première loi d'assistance sociale apparaisse : l'AMG (aide médicale gratuite). Aboutira à la fin du XIXème siècle, le 09/04/1898, la première loi relative aux accidents du travail : obligation de réparer puis de compenser les atteintes liées au travail.

Parallèlement à cela, en 1830 il y a l'invention de la sonographie (écriture par traits et points) par Charles Barbier de la Serre, puis le Braille par Louis Braille (1809-1852) né aveugle qui fut professeur de l'Institut royal des aveugles.

Pour les sourds-muets, l'importation des signes français aux Etats-Unis en 1816 par Laurent Clerc, Ferdinand Berthier (professeur sourd, doyen des professeurs sourds de l'Institut de Paris de 1840 à 1850) va introduire la notion de peuple et de culture sourde, espère un langage universel, et défendra le bilinguisme [4]. En 1834, la société centrale des sourds-muets de Paris est créée, puis la première Fédération Nationale des Sourds muets par Joseph Cochefer en 1880, grand ami de Paul Deshanel (1855-1893).

Pourtant, des évènements, qui vont bousculer et contrarier ces avancées, car nous sommes dans une société où l'homme croit qu'il va résoudre tous les problèmes y compris celui des infirmes, vont suivre :

- les expériences douloureuses et cruelles du Docteur ITARD (1774-1839) sur les sourds-muets, que l'on connaît plus par son ouvrage sur l'enfant sauvage de l'Aveyron ;

- le Congrès de Milan, en 1880, qui aura pour conséquence 
l'interdiction de la langue des signes (participaient à ce congrès 164 entendants et 2 sourds). On prône l'oralisation à tout prix;

- la Loi sur l'école obligatoire, par Jules Ferry en 1881 et 1882, qui isolera ceux qui ne pourront être acceptés dans une école dite standardisée ;

- au XIXème siècle, ce sont aussi le développement des cirques, foires où l'on exhibait toutes sortes d'individus handicapés, devant un public curieux. Pourtant, ils se défendaient en disant qu'ils gagnaient leur vie et ne dépendaient d'aucune charité publique [6] ;

- en 1880, la notion d'Eugénisme apparaît, introduite par François Galton, selon qui il fallait favoriser la reproduction des " bons " sujets et ralentir, voire stopper, la reproduction des " mauvais " sujets, en voulant préserver les races les « mieux douées » [6] ; cette nouvelle notion va faire boule de neige et basculer vers le $X X X^{\text {ème }}$ siècle.

\section{Le XXème siècle et le début du XXIème siècle}

C'est le siècle des lois pour les personnes en situation de handicap, mais c'est aussi la création de la sécurité sociale, les différentes associations pour défendre les personnes en situation de handicap, ceci découlant de la première guerre mondiale, de la tuberculose, de la poliomyélite :

- Loi du 30/03/1919 : loi de création des pensions aux mutilés et victimes de guerre, centres de rééducation, d'appareillage, et des emplois réservés qui sera renforcée par la loi suivante,

- loi du 26/04/1924 assurant un emploi obligatoire des mutilés avec un quota de $10 \%$ dans les entreprises de plus de 10 salariés, ce taux passera à 6\% en 1991 dans les entreprises de 20 salariés, avec un versement à l'AGEFIPH (Association nationale de Gestion pour l'Insertion Professionnelle des Handicapés) si ce taux n'est pas atteint. En 1930 extension de cette loi aux mutilés du travail ;

- le 04/10/1945, ordonnance de la Sécurité Sociale (88 articles);

- en 1954, création des CAT (Centres d'aide par le travail) ;

- loi du 23/11/1957 [7] sur le reclassement des travailleurs handicapés (première apparition du mot handicapé), création des ateliers protégés, les handicapés mentaux sont inclus dans le reclassement des travailleurs handicapés, création des $\mathrm{CDOI}$ (commission départementale d'orientation des infirmes);

- création de mesures de protection judiciaires (curatelle et tutelle) le 03/01/1968 ;

- le 13/07/1971, création de l'AAH (Allocation aux adultes handicapés), aujourd'hui elle est de 621,27 euros par mois ;

- la loi d'orientation en faveur des personnes handicapées du $30 / 06 / 1975$ [8], renforce les autres lois, et les CDOI sont remplacés par la COTOREP pour les adultes (Commission Technique d'Orientation et de Reclassement Professionnel) et la CDES (Commission Départementale d'Education Spéciale) pour les enfants. Création du CNCPH (Conseil National Consultatif des Personnes Handicapées) qui a pour mission d'assister l'Etat dans la coordination de toutes les actions en vue de satisfaire à l'obligation nationale, que sont « la prévention et le dépistage des handicapés, les soins, l'éducation, la formation et l'orientation professionnelle, l'emploi, la garantie d'un minimum de ressources, l'intégration sociale et l'accès aux sports, aux loisirs du mineur et de l'adulte handicapés physiques, sensoriels ou mentaux " ;

- loi du 07/01/1981 sur la protection de l'emploi des salariés victimes d'un accident du travail ou d'une maladie professionnelle ;

- le 10/07/1987 [9], loi relative à l'obligation d'emploi des travailleurs handicapés reconnus par la COTOREP, maladie professionnelle ou accidents du travail dont l'incapacité dépasse $10 \%$, cette obligation d'emploi est étendue au secteur public ;

- le 13/01/1989, amendement Créton : selon l'article 22, obligation de maintenir les enfants dans des institutions d'éducation spécialisées au-delà de l'âge réglementaire (si il n'y a pas de place dans les établissement adultes) ;

- loi du 12/07/1990 [10] relative à la protection des personnes contre la discrimination en raison de leur état de santé ou de leur handicap ;

- loi du 13/07/1991 [11], suivie du décret du 26/01/1994 sur l'accessibilité des locaux d'habitation, des lieux de travail, des locaux recevant du public aux personnes handicapées, puis de l'arrêté du 31/08/1999 sur la prescription technique pour l'accessibilité de la voirie ;

- loi du 31/12/1992 : l'Etat oblige à rechercher une possibilité de reclassement à tout salarié devenu inapte physiquement;

- loi du 04/07/1996, Circulaire n 96-428 sur la prise en charge et la réinsertion sociale et professionnelle des traumatisés crâniens.

Enfin, le XX|ème siècle commence très fort, avec de nouveau une nouvelle loi, précédée de quelques lois et création de divers organismes :

- loi du 17/01/2002 [12] sur la modernisation sociale ;

- le 30/12/2004 [13] création de la haute autorité de lutte contre les discriminations et pour l'égalité, HALDE ;

- création en 2004 de la CNSA (caisse nationale de solidarité pour l'autonomie) dont la mission sera renforcée par la loi du $11 / 02 / 2005$, avec trois missions : financer les aides en faveur des personnes âgées dépendantes et des personnes handicapées ; garantir l'égalité de traitement sur tout le territoire pour l'ensemble des handicaps ; assurer une mission d'expertise, d'information et d'animation pour suivre la qualité du service rendu aux personnes.

- et enfin, la loi du 11/02/2005 sur l'égalité des droits et des chances, la participation et la citoyenneté des personnes handicapées [14]. Les grands principes de cette nouvelle loi repose ni plus ni moins sur celle de 1975 en notant toutefois quelques modifications.

\section{- Le droit à compensation.}

Création de la prestation de compensation du handicap ( $\mathrm{PCH}$ ) remplaçant l'ACTP (allocation compensatrice tierce personne) et l'AFP (allocation frais professionnels), elle est basée maintenant sur 5 grands types d'aide, des aides humaines, techniques, aménagements du logement et du véhicule ainsi que des surcoûts de transport, des aides animalières, et enfin des aides exceptionnelles et spécifiques (Tableaux 1, 2, 3 et 4).

Petite ineptie sur les aides humaines qui concernent les actes 
Tableau 1 : Eléments de la prestation de compensation : montants, durées, tarifs - tarifs au 01/01/2006.

\begin{tabular}{|c|c|c|c|c|c|}
\hline \multicolumn{2}{|c|}{ Elément de la prestation de compensation } & \multirow{6}{*}{$\begin{array}{l}\text { Montant maximal attribuable } \\
\text { Montant maximal mensuel : Egal au tarif } \\
\text { horaire le plus élevé multiplié par la } \\
\text { durée quotidienne maximale fixée par le } \\
\text { référentiel figurant à l'annexe } 2-5 \text { du } \\
\text { CASF, multiplié par } 365 \text { et divisé par } \\
\text { 12.** }\end{array}$} & Durée maximale* & \multicolumn{2}{|l|}{ Tarif } \\
\hline \multirow{6}{*}{\multicolumn{2}{|c|}{$\begin{array}{l}1^{\Theta r} \text { élément } \\
\text { aides humaines }\end{array}$}} & & \multirow[t]{6}{*}{10 ans } & Statut de l'aidant & Tarif horaire \\
\hline & & & & Emploi direct & $11,02 € / h$ \\
\hline & & & & Service mandataire & $12,12 € / h$ \\
\hline & & & & Service prestataire & $14,43 € / h$ \\
\hline & & & & Dédommagement & $3,10 € / h$ \\
\hline & & & & $\begin{array}{l}\text { Dédommagement (si renoncement total ou } \\
\text { partiel à une activité professionnelle) }\end{array}$ & $4,64 € / h$ \\
\hline \multirow[t]{2}{*}{$\begin{array}{l}2^{\text {én̄è }} \text { élément } \\
\text { aides techniques }\end{array}$} & régie générale & $3960 €$ & \multirow[t]{2}{*}{3 ans } & \multirow{2}{*}{\multicolumn{2}{|c|}{$\begin{array}{l}\text { Selon les aides techniques : tarif détaillé ou } 75 \% \text { du prix dans } \\
\text { la limite du montant maximal attribuable }\end{array}$}} \\
\hline & $\begin{array}{l}\text { si une AT, et le cas échéant, ses } \\
\text { accessoires, sont tarifés à au moins } \\
3000 €\end{array}$ & $\begin{array}{l}3960+\text { montant des tarifs de cette AT + } \\
\text { montant des accessoires - tarif LPP }\end{array}$ & & & \\
\hline \multirow{2}{*}{$\begin{array}{l}3^{\text {eme }} \text { élément } \\
\text { aménagement } \\
\text { du logement, du } \\
\text { véhicule et } \\
\text { surcoút lies aux } \\
\text { transports }\end{array}$} & logement & $10000 €$ & 10 ans & \multicolumn{2}{|l|}{$\begin{array}{l}\text { Tranche de } 0 \text { à } 1500 €: 100 \% \\
\text { Tranche au-delà de } 1500 €: 50 \% * * * \\
\text { Déménagement : } 3000 \text { euros }\end{array}$} \\
\hline & véhicule, surcoût lié aux transports & $5000 €$ passe a $1200 €$ & 5 ans & $\begin{array}{l}\text { Véhicule : tranche de } 0 \text { à } 1500 €: \\
\text { Véhicule : tranche au-delà de } 1500 € \text { : } \\
\text { Transport }\end{array}$ & $\begin{array}{l}100 \% \\
75 \% \%^{* * \star} \\
75 \%\end{array}$ \\
\hline \multirow{2}{*}{$\begin{array}{l}4^{\text {enere }} \text { élément } \\
\text { charges } \\
\text { spécifiques et } \\
\text { exceptionnelles }\end{array}$} & charges spécifiques & $100 €$ /mois & 10 ans & \multicolumn{2}{|c|}{$\begin{array}{l}\text { Selon les produits : tarif détaillé ou } 75 \% \text { du coût dans la limite } \\
\text { du montant maximal attribuable }\end{array}$} \\
\hline & charges exceptionnelles & $1800 €$ & 3 ans & \multicolumn{2}{|c|}{$75 \%$ du prix dans la limite du montant maximal attribuabie } \\
\hline \multicolumn{2}{|c|}{$\begin{array}{l}5^{6 m e} \text { élément aide régie générale } \\
\text { animalière }\end{array}$} & $3000 €$ & 5 ans & \multicolumn{2}{|l|}{ Si versement mensuel : 50 euros $/$ mois } \\
\hline \multicolumn{6}{|c|}{$\begin{array}{l}\text { *Durée maximale: } \\
\text { - Durée maximale d'attribution lorsque la prestation de compensation doit faire f'objet d'un versement mensuel, } \\
\text { - En cas de versements ponctuels, le total des versements comespondant à chaque élément de la prestation de compensation ne peut dépasser les montants maximums prévus a } \\
\text { l'Article } R .245-37 \text { sur une péniode ne dépassant pas la dunée maximale d'attribution de l'élément. }\end{array}$} \\
\hline
\end{tabular}

DGAS Phan - févier 2006

Tableau 2 : Tarifs horaires applicables au 1er élément de la prestation de compensation (aides humaines) - tarifs au 01/01/2006.

\begin{tabular}{|c|c|c|c|}
\hline \multirow{2}{*}{$\begin{array}{l}\text { Modalité de l'aide humaine } \\
\text { Emploi direct }\end{array}$} & \multirow{2}{*}{$\begin{array}{l}\text { Tarif horaire } \\
\text { applicable pour la } \\
\text { prestation } \\
11,02 € / \mathrm{h}\end{array}$} & \multicolumn{2}{|c|}{ Modalité de calcul, convention de référence et salaire horaire de référence } \\
\hline & & $\begin{array}{l}130 \% \text { du salaire horaire brut sans ancienneté d'une assistante de vie } \\
\text { pour personne dépendante de niveau 3, au sens de la convention } \\
\text { collective nationale des salariés du particulier employeur du } 24 \text { novembre } \\
1999\end{array}$ & $8,48 € h$ \\
\hline Service mandataire & $12,12 € / h$ & Majoration de $10 \%$ du tanif emploi direct & $8,48 \in h$ \\
\hline Service prestataire & $14,43 \in / h$ & $\begin{array}{l}145 \% \text { du salaire horaire brut d'une auxiliaire de vie ayant moins d'un an } \\
\text { d'ancienneté, au sens de l'accord de la branche aide à domicile du } 29 \\
\text { mars } 2002 \text { relatif aux emplois et rémunérations (catégorie C, indice } 295 \text {, } \\
\text { valeur du point : } 5,115 \text {, modalité de calcul du salaire horaire: } \\
295^{*} 5,115 / 151,67=9,95 \text { ) }\end{array}$ & $9,95 € h$ \\
\hline Dédommagement d'un aidant familial & $3,10 € / h$ & $50 \%$ du SMIC horaire net & $6,19 € / h$ \\
\hline $\begin{array}{l}\text { Dédommagement d'un aidant familia } \\
\text { cesse ou renonce totalement ou partielle } \\
\text { une activité professionnelle }\end{array}$ & $4,64 € / h$ & $75 \%$ du SMIC horaire net & $6,19 \in \mathrm{g}$ \\
\hline
\end{tabular}

Le montant maximum du dédommagement mensuel de chaque aidant familial est de 85\% du SMIC mensuel net applicable aux emplois familiaux, calculé sur la base de 35 heures par semaine, soit $798 \epsilon$ par mois.

DGAS Phan - févier 2006 
Tableau 3 : Nombre d'heures et montant du 1er élément (aides humaines) pour des situations particulières (art. D.245-9 du CASF) au 01/01/2006.

\section{Situation particulière}

Nombre d'heures Tarif horaire attribuées par mois applicable

\section{Montant}

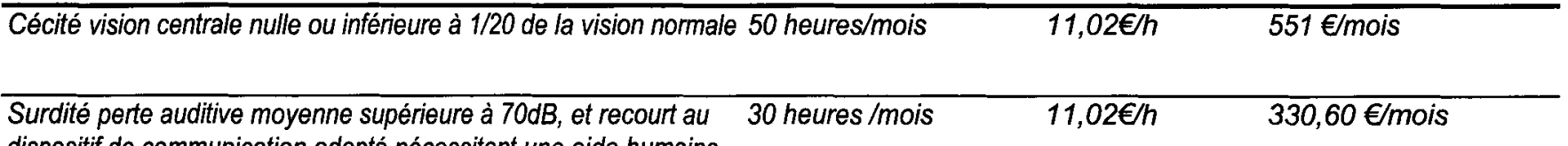
dispositif de communication adapté nécessitant une aide humaine

DGAS Phan - fevrier 2006

Tableau 4 : Temps plafonds pour le 1er élément (aides humaines).

\begin{tabular}{|c|c|c|}
\hline Domaines & Activités & Temps plafonds * \\
\hline \multicolumn{3}{|c|}{ ents dans le 5 heures par jour } \\
\hline & $\begin{array}{l}\text { Déplacements à l'extérieur exigés par des démarches liées au handicap de la } \\
\text { personne et nécessitant la présence personnelle }\end{array}$ & 30 heures par an \\
\hline & Participation a la vie sociale & 30 heures par mois \\
\hline \multirow[t]{2}{*}{ Surveillance } & $\begin{array}{l}\text { Les personnes qui s'exposent à un danger du fait d'une altération d'une ou plusieurs } \\
\text { fonctions mentales, cognitives ou psychiques. }\end{array}$ & 3 heures par jour \\
\hline & $\begin{array}{l}\text { Les personnes qui nécessitent à la fois une aide totale pour la plupart des actes } \\
\text { essentiels et une présence due à un besoin de soins constants ou quasi constants. }\end{array}$ & $\begin{array}{l}12 \text { heures par jour, (cumul actes essentiels } \\
\text { et surveillance) }\end{array}$ \\
\hline
\end{tabular}

Frais supplémentaires liés a l'exercice d'une activité professionnelle ou d'une fonction élective

156 heures par an

* Dans des situations exceptionnelles, la CDA peut porter le temps d'aide attribué au titre des actes essentiels ou de la surveillance au-delà des temps plafonds.

DGAS Phan - fërier 2006

Tableau 5 : Indicateurs.

Indicateur de KATZ (ADL : Activities of Daily Living)

Indicateur KATZ Besoin d'aide (les 7 premiers ètant de gravité croissante)

A
B
C
D
E
F
G
H

Indépendants pour les six activités

Dépendant pour une seule activité des 6 activités

Dépendant pour 2 des 6 activités dont la première

Dépendant pour 3 des 6 activités, dont les 2 premières

Dépendant pour 4 activités dont les 3 premières

Dépendant pour 5 activités dont les 4 premières

Dépendant pour les 6 activités

Dépendant pour au moins deux activités,

sans être classable en $C, D, E$ ou $F$

Critères : 1 . faire sa toilette.

2. s'habiller.

3. aller aux toilettes et les utiliser.

4. se coucher ou quitter son lit et

s'asseoir ou quitter son siège.

5. contrôler ses selles et ses urines.

6. manger des aliments déjà

préparés (cuisinés et coupés).

0 . sortir du domicile sans aide.

\section{Indicateur de Colvez}

1. Confiné au lit ou au fauteuil non roulant

2. Non classé en 1, mais ayant besoin d'aide pour la toilette et l'habillage

3. Non classé en 1 ou 2, mais ayant besoin d'aide pour sortir de l'institution

4. Autres cas

Indicateur Incapacité fondé sur la mobilité

1. Ne peut sortir de son domicile sans aide

2. Ne sort plus de son domicile

3. Confiné au lit ou au fauteuil

D'après l'enquête HID Juillet 2001 
essentiels (Tableau 4). Ils émanent très certainement des indicateurs de Katz (Activities of Daily Living) et de Colvez ainsi que des Indicateurs Incapacité fondé sur la mobilité (Tableau 5).

Prenons un exemple concret : madame $X$, paraplégique en D7 à la suite d'un accident de la route, fait la demande de $\mathrm{PCH}$. Elle sort d'une année de rééducation en centre spécialisé (ergothérapeute, kinésithérapeute, etc...) qui lui ont appris à être autonome. Elle sait par exemple s'habiller, faire sa toilette, se sonder, faire un transfert de fauteuil, possède son permis de conduire et sait parfaitement maîtriser le rangement de son fauteuil dans la voiture. A-t-elle besoin d'aides humaines telles que définis par la nouvelle loi ? NON, et heureusement puisque le but est de pouvoir redonner le plus d'autonomie possible à la personne en situation de handicap dans les centres. Mais ne manque-t-il pas quelque chose de très important ? Et OUI, que fait-on des tâches ménagères (poussières, ordures, lavage et surtout repassage en sachant que les tables à repasser, les fers à repasser sont très lourds, à moins d'avoir les moyens de s'offrir une presse à repasser. Passer l'aspirateur et la serpillière est quasi impossible pour cette personne !!!!!). Cette personne se retrouvera donc sans aides humaines ...

- Le droit à la scolarisation des enfants en situation de handicap ainsi que le droit au travail en milieu ordinaire.

Déjà existant mais renforcé au niveau des amendes si les entreprises ne font aucun effort pour embaucher des personnes en situation de handicap. A noter pour ce qui est de l'emploi, 24\% de la population active des personnes en situation de handicap est au chômage ; dans les études supérieures, seulement $10 \%$ des étudiants en situation de handicap sont des sourds.

\section{- L'accessibilité.}

La mise en accessibilité de toute la chaîne de déplacement (aménagement de la voirie, accès aux gares, transport en commun...) afin de pouvoir accéder sans rupture aux bâtiments recevant du public, qui eux-mêmes doivent pouvoir donner les différentes informations et rendre accessible leurs locaux pour tous les types de handicap (physique, sensoriel, mental, cognitif, polyhandicap). Ce délai est fixé à 5 ans pour les préfectures et les universités, et à 10 ans pour le reste. Des sanctions sont susceptibles en cas de non respect du délai (fermeture, amende, remboursement des subventions publiques...). Pour les transports, un délai de 3 ans est donné en cas d'impossibilité technique avérée de mise en accessibilité d'un système de transport, afin de mettre à disposition des moyens de substitution accessibles au même tarif que les transports collectifs. La formation des architectes à l'accessibilité est devenue obligatoire.

\section{- Enfin les Maisons départementales du handicap (MDPH) remplace les CDES/COTOREP.}

Les MDPH associent toutes les compétences impliquées actuellement dans l'accompagnement des personnes handicapées : le conseil général, la direction départementale des affaires sanitaires et sociales (DDASS), la direction départementale du travail, de l'emploi et de la formation professionnelle (DDTEFP), les caisses d'allocations familiales (CAF), les caisses primaires d'assurance maladie (CPAM) et l'inspection académique. D'autres organismes peuvent être associés. Certes, c'est un endroit où tout se trouve centralisé mais où le délai d'attente lorsque la personne remet son dossier est de l'ordre de 7 à 10 mois (délai d'attente identique à l'ancienne organisation).
Les différents articles qui suivent intéressent particulièrement notre profession à savoir une définition du handicap (la première), la formation des professionnels de santé et l'accessibilité.

- Définition du handicap, Art L. 114.

Constitue un handicap, toute limitation d'activité ou restriction de participation à la vie en société subie dans son environnement par une personne en raison d'une altération substantielle, durable ou définitive, d'une ou plusieurs fonctions physiques, sensorielles, mentales, cognitives ou psychiques, d'un polyhandicap ou d'un trouble de santé invalidant ("Sont inadaptés à la société dont ils font partie, les enfants, les adolescents et les adultes qui, pour des raisons diverses, plus ou moins graves, éprouvent des difficultés, plus ou moins grandes, à être et à agir comme les autres (..) Ces individus sont « handicapés parce qu'ils subissent par suite de leur état physique, mental, caractériel ou de leur situation sociale, des troubles qui constituent pour eux des handicaps "Bloch-Lainé F., "Étude du problème général de l'inadaptation des personnes handicapées, Rapport présenté au Premier ministre", Paris, 1967).

Je rajouterai cette autre définition du handicap d'après la Classification internationale des handicapés, qui définit ainsi : « toute personne souffrant d'une déficience - aspect lésionnel - ou d'une incapacité - aspect fonctionnel - qui limite ou interdit toutes activités considérées comme normales pour un être humain ".

\section{- Sur la formation.}

Les professionnels de santé et du secteur médico-social reçoivent, au cours de leur formation initiale ou continue, une formation spécifique concernant l'évolution des connaissances relatives aux pathologies à l'origine des handicaps et les innovations thérapeutiques, technologiques, pédagogiques, éducatives et sociales les concernant, l'accueil et l'accompagnement des personnes handicapées, ainsi que l'annonce du handicap.

\section{- Sur l'accessibilité, Art L.111-7-1.}

Les dispositions architecturales, les aménagements et équipements intérieurs et extérieurs des locaux d'habitation, qu'ils soient la propriété de personnes privées ou publiques, des établissements recevant du public, des installations ouvertes au public et des lieux de travail doivent être tels que ces locaux et installations soient accessibles à tous, et notamment aux personnes handicapées, quel que soit le type de handicap, notamment physique, sensoriel, cognitif, mental ou psychique, dans les cas et selon les conditions déterminés aux articles $L$. 111-7-1 à L. 111-7-3. Ces dispositions ne sont pas obligatoires pour les propriétaires construisant ou améliorant un logement pour leur propre usage.

Est considéré comme accessible aux personnes handicapées tout bâtiment ou aménagement permettant, dans des conditions normales de fonctionnement, à des personnes handicapées, avec la plus grande autonomie possible, de circuler, d'accéder aux locaux et équipements, d'utiliser les équipements, de se repérer, de communiquer et de bénéficier des prestations en vue desquelles cet établissement ou cette installation a été conçu. Les conditions d'accès des personnes handicapées doivent être les mêmes que celles des personnes valides ou, à défaut, présenter une qualité d'usage équivalente.

Autre définition de la situation de handicap : toute personne se trouvant à un instant $t$ de sa vie devant une situation de handicap, c'est-à-dire ne pouvant marcher, communiquer, voir, etc.... par 
manque de moyens mis à sa disposition lui permettant d'être autonome. C'est une nouvelle notion qui commence à émerger : tout le monde peut être en situation de handicap à un moment de sa vie, exemples :

- Jacques (Figure 1) revient des sports d'hiver avec une jambe cassée et habite au 3 ème étage seul sans ascenseur,

- Ludovic (Figure 2), paraplégique à la suite d'un accident de la voie publique il y a 4 ans, habite un immeuble au rez-dechaussée, appartement qu'il a fait adapter à sa situation de handicap, et conduit sa voiture équipée.
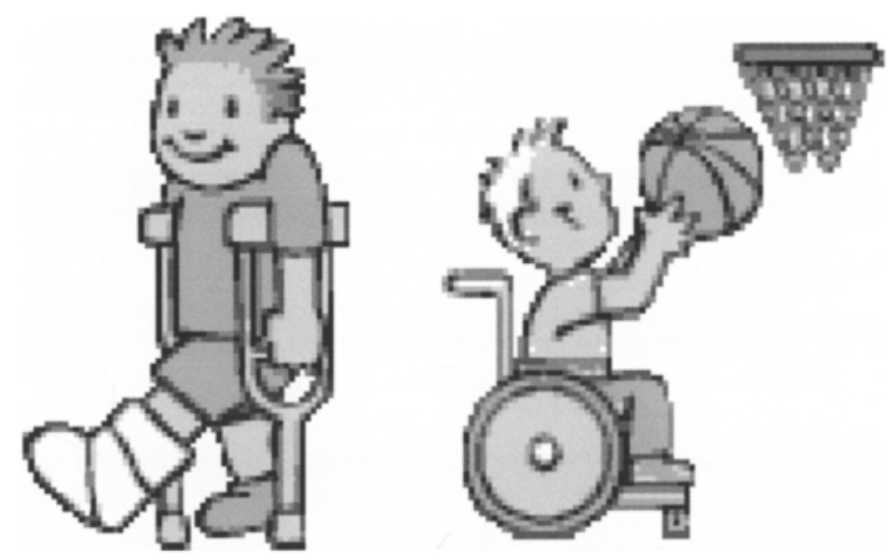

Figures 1 et 2: Jacques revient des sports d'hiver avec une jambe cassée. Ludovic, paraplégique à la suite d'un accident de la voie publique il y a 4 ans.

Qui est le plus en situation de handicap ?

D'après G. Ehretsmann, ergothérapeute « C'est l'organisation de nos sociétés qui provoque une situation de handicap en ne prenant pas en compte les besoins spécifiques de tous nos concitoyens " et l'OMS, qui réfléchit sur le problème depuis 1980 , a révisé la définition : la $\mathrm{CIH}$ (classification internationale du handicap) est remplacée par la CIF (classification internationale des fonctionnalités). Depuis 2002, I'IRIT (Institut de recherche en informatique de Toulouse) est le coordonnateur français d'un réseau de la commission européenne design for all (conception pour tous). II s'agit de développer des systèmes utilisables aussi bien par les personnes dites valides que pour les personnes handicapées ou âgées, dans toutes les situations où la mobilité, la perception (vue, ouie, etc...), la cognition ou le dialogue, sont altérés. Bref, une vaste réflexion sur l'être humain en fonction de la situation dans laquelle il se trouve [17]. En effet, quelques chiffres viennent corroborer cette situation.

\section{QUELQUES CHIFFRES}

En France, il y aurait 3 à 4 millions de personnes en situation de handicap officiel (CDES, COTOREP, maintenant MDPH), mais cela varie suivant la définition de 1,8 million à 22,5 millions (ce dernier chiffre émane d'une enquête HID, Handicaps Incapacités Dépendance, faite par l'INSEE entre 1998 et 2002, incluant les plus de 60 ans) [16].

Cela représente près de $40 \%$ de la population qui déclare avoir une situation de handicap ou une gène dans la vie quotidienne.

Dans un communiqué de presse en date du 09/02/2006, Philippe
Bas (ancien ministre délégué à la sécurité sociale, personnes âgées, personnes handicapées, familles) donnait comme chiffre 5 millions de personnes en situation de handicap [1].

En Europe cela représente plus de 40 millions de personnes. A noter que d'ici 2050 , la population des plus de 85 ans va quintupler en France, nous sommes tous condamnés à être un jour dans une situation de handicap.

\section{COMMENTAIRES}

A en croire toutes les dispositions prises par les différentes lois, les personnes en situation de handicap n'auraient plus de problèmes de nos jours et pourtant. .. La majorité des sourds après les années 50 se trouvent sous éduqués. En 1967, un médecin américain crée le LPC (langage parlé complété), on lève peu à peu l'interdit dans les écoles de la langue des signes, il sera officialisé en 1975 comme la LSF (langue des signes française) mais ce n'est seulement qu'en 1991, grâce à Laurent Fabius, que l'éducation des enfants sourds pourra se faire en langue des signes. La première école d'interprète ouvre en 1980 et le $17 / 06 / 1988$, le parlement européen reconnaît la langue des signes dans chaque état membre. Dans les années 90 (1993) les sourds se voient imposer l'implant cochléaire sans résultat probants (rapport du Comité Consultatif National d'Ethique). Le rapport Gillot de 1998 dit ceci "pour que le siècle qui s'ouvre devant nous fasse du droit des sourds le devoir des entendants ", malheureusement seulement $12 \%$ des émissions télévisées sont sous titrées, une émission " l'œil et la main " sur France 5 , trois fois par mois, et seulement 120 interprètes en France. La loi du 11/02/2005 reconnaît enfin la LSF comme langue officielle des sourds et peut être enseignée, ce qui va leur permettrent grâce, à la $\mathrm{PCH}$, d'obtenir des heures d'interprétariat pour les différentes démarches sociales. Là encore, une injustice sur le nombre d'heures d'aides humaines dans des situations particulières : 50 heures/mois pour les aveugles, 30 heures/mois pour les sourds ayant recourt au dispositif de communication adapté nécessitant une aide humaine (Tableau 3 ).

La loi du 04/07/1942 interdisait aux professeurs aveugles d'exercer dans le secondaire, en 1964 les professeurs aveugles sont aptes à enseigner les mathématiques, en 1967 les sciences économiques et sociales ainsi que l'histoire géographie. Près de $90 \%$ des sites Internet sont inaccessibles aux non voyants. II faudra attendre 1989 pour que la fédération internationale des écoles de chiens guide d'aveugles soit reconnue officiellement alors que cette idée est née en 1917 en Allemagne, et ce n'est qu'en 1951 qu'elle apparaît en France par Paul Coterville. Une émission une fois par mois sur France 5 " A vous de voir ".

La majorité des bâtiments recevant du public (notamment les hôpitaux), les transports, les loisirs, le tourisme sont inaccessibles pour les personnes handicapées en fauteuil. On notera ici que le handicap moteur n'a aucune émission télévisée fait par lui et parlant de lui, la raison ? ...

Autre fait, ou lorsque l'humiliation est portée à son comble : devoir rendre des comptes sur l'usage que l'on fait de nos différentes aides (l'ACTPIAFP ou la PCH) au moyen de justificatifs ou d'attestation sur l'honneur, le summum en vient de devoir rembourser les sommes déjà versées lorsque l'on revient à meilleure fortune (sauf fait nouveau, la PCH article L.245-7 du code de l'action sociale et des familles) !!! Je ne dirai certainement rien à ce sujet, si ce n'est que pour la plupart des aides que l'on donne (allocation de rentrée scolaire, allocation familiales etc..), 
à ma connaissance aucun justificatif n'est demandé quant à la bonne utilisation de ces deniers... !!!

Dans une société qui prône sans cesse la rentabilité, la performance, le beau, la perfection, n'y a $t$ il pas dans ces lois une bonne conscience du législateur à vouloir mettre noir sur blanc des lois que nombre de personnes ignorent, ou qui ne sont pas appliquées ?

A commencer par les sourds. En effet les sourds ne s'identifient pas par rapport aux personnes normales mais par rapport aux entendants, pour eux la surdité n'est pas une situation de handicap, (bien qu'ils possèdent tous la carte d'invalidité à $80 \%$, ceci leur permettant d'avoir l'AAH car beaucoup d'entre eux ne trouvent pas de travail), mais un monde à part avec sa culture, ses règles, sa langue, sa syntaxe, son humour aussi. Yves Delaporte dit ceci « La loi entendante dit ce que sont les sourds, et ce qu'il faut en faire. Elle dit aussi comment il faut les nommer. L'histoire de la dénomination des sourds est le reflet de la manière dont on les a regardés, et dont on les a traités (...). Le gouvernement qui décide, les médecins qui conseillent, les familles qui leur obéissent, tous sont « complices » (...). Les médecins sont la figure emblématique des conflits culturels entre sourds et entendants. Ce sont les plus cultivés des entendants, les plus diplômés, ceux qui sont payés pour savoir, ceux que le monde entendant délègue auprès des sourds pour les soigner, et ce sont notoirement ceux qui en savent le moins et se méprennent le plus sur eux. (...) (Traduction en langue des signes : Des sourds, les docteurs ne savent voir que leurs oreilles)." [3].

Cette dernière phrase pourrait tout aussi bien s'adapter pour toutes les personnes en situation de handicap car quelles sont les personnes que nous voyons en premier si ce n'est les médecins ? Les médecins n'ont-ils pas pour vocation de soigner et de guérir ? Le patient vient voir le médecin pour un problème $X$, le médecin le soigne, le guérit, le patient le remercie et on retourne à l'état antérieur. Mais à partir du moment où il y a impossibilité de guérison, voire une situation de handicap, le médecin reste face à son patient comme ne sachant plus quoi dire, ou faire, il pense à tort qu'il est dans une situation d'échec. Ce qu'il ignore, c'est que la personne en situation de handicap pallie à ses manques et que parfois elle est même capable d'accomplir des choses qui pour des gens " normaux " parait extraordinaire (aspect positif) ou irraisonnable (aspect négatif) ; deux exemples : on s'émerveille devant des athlètes paraplégiques accomplissant une course de fauteuils à des vitesses incroyables, et l'on va s'inquiéter d'une patiente paraplégique désirant une grossesse quant à l'avenir de son futur enfant, contradiction qui est en plus accentuée par un vide de la part des législateurs qui ne parle pas de la parentalité des personnes en situation de handicap (la nouvelle loi et les lois antérieures ont esquivées ce sujet). Si en effet tout est prévu pour l'arrivée d'un enfant en situation de handicap avec des parents " normaux ", rien sur la maternité des personnes en situation de handicap, oubli ou gommage volontaire de la part des hommes politiques ? II ne faut donc pas s'étonner aujourd'hui encore que l'on refuse des patientes en situation de handicap enceintes dans certains hôpitaux, et que la plupart des hôpitaux, pour ne pas dire tous ne sont pas accessibles au sens propre de la définition de la loi du 11/02/2005. Le matériel de puériculture adapté pour les personnes en situation de handicap est inexistant à l'échelle industrielle (il faut compter 3000 euros pour un lit pour nouveau-né adapté pour une mère ou un père atteint d'une paraplégie, dixit Marie Ladret, ergothérapeute).
C'est pourquoi nous avons instauré à l'IMM (Institut Mutualiste Montsouris) dans le Département Mère-Enfant, dirigé par le Dr Henri Cohen, une consultation tous les vendredis matin avec évaluation des situations de handicap de chaque future maman, cette consultation étant faite en LSF si nécessaire. La prise en charge des patientes après accord du staff (nous sommes une maternité de niveau 1) se fait conjointement avec un gynécologue obstétricien (Dr Madeleine Azarian Nazac) et une sage-femme, parallèlement et conjointement à cela au niveau de l'AMP une consultation handicap-stérilité par le Dr Vincent Izard, un travail débutant avec ESCAVIE (Espace Conseil pour l'Autonomie en milieu ordinaire de Vie) avec une ergothérapeute, Marie Ladret, et un assistant social, Frank Berton, afin de préparer le retour à la maison (déplacement au domicile des futurs parents). Une fiche de situation de handicap (document fiche) pour le personnel soignant est remplie par la patiente, et la mise en accessibilité d'une chambre et d'une pièce de consultation (Figures $3,4,5$, 6) (avec des moyens restreints, car pour l'instant nous n'avons pas obtenu de la part de la direction qu'elle réalise complètement une chambre adaptée), et d'une pièce de consultation avec un fauteuil adapté pour personne en situation de handicap moteur. Pour l'avenir nous réfléchissons aux différentes adaptations pour le handicap sensoriel.

II est vrai que cette démarche ne peut se faire sans une volonté réelle de la part de toute une équipe, et nous nous heurtons encore à quelques difficultés individualistes. De même nous ne souhaitons faire une ghettoïsation des situations de handicap, et ce ne sera jamais un lieu de spécificité ni de spécialité. Peutêtre l'envie d'un autre regard, ou tout simpiement l'envie de le faire sans porter aucun jugement et simplement le bonheur de voir un couple se réaliser pleinement dans son rôle de parents, malgré la situation de handicap de l'un deux ou des deux.

Enfin je terminerai par un sujet épineux sujet qui vous concerne: les maladies génétiques et la loi de bioéthique de 2004. II faut savoir qu'au XXème siècle des prix Nobel et des hommes politiques ont perpétré l'eugénisme prôné au XIXème siècle par Galton. Ainsi Richet en 1919 dans la " Sélection humaine ", Alexis Carrel en 1935 dans "L'homme cet inconnu ». Des campagnes de stérilisation chez des sujets dits arriérés vont proliférer. Et l'on ne s'étonnera pas qu'en 1939 le nazisme la mette en application et tue le premier nouveau-né malformé pour transformer cela ensuite en génocide [6]. Dans une déclaration de Francis Crick, citée par P Thuillier en 1984, « Aucun enfant nouveau-né ne devrait être reconnu humain avant d'avoir passé un certain nombre de tests portant sur la dotation génétique. S’il ne réussit pas ces tests, il perd son droit à la vie ". N'oublions pas non plus l'arrêt Perruche du 27/11/2000.

Que penser quand on entend des hommes politiques dire de nos jours que " permettre à un enfant handicapé de venir au monde est une faute parentale et peut-être même le témoignage d'un égoïsme démesuré " , et qu'il aura fallu pour éviter tout débordement une loi votée définitivement le 04/03/2002 « nul, fût-il né handicapé, ne peut se prévaloir d'un préjudice du seul fait de sa naissance »[6].

Les médias vont aussi accentuer cette image néfaste de la personne en situation de handicap, de la malformation, en instituant une idée que les parents désirent avant tout un bébé parfait. La technologie peut non seulement détecter la malformation mais aussi éliminer l'enfant à naitre [6] ; de même, au risque d'être choquante, nous ne voyons jamais à la télé les fauteuils roulants des quelques peu nombreux présentateurs en 


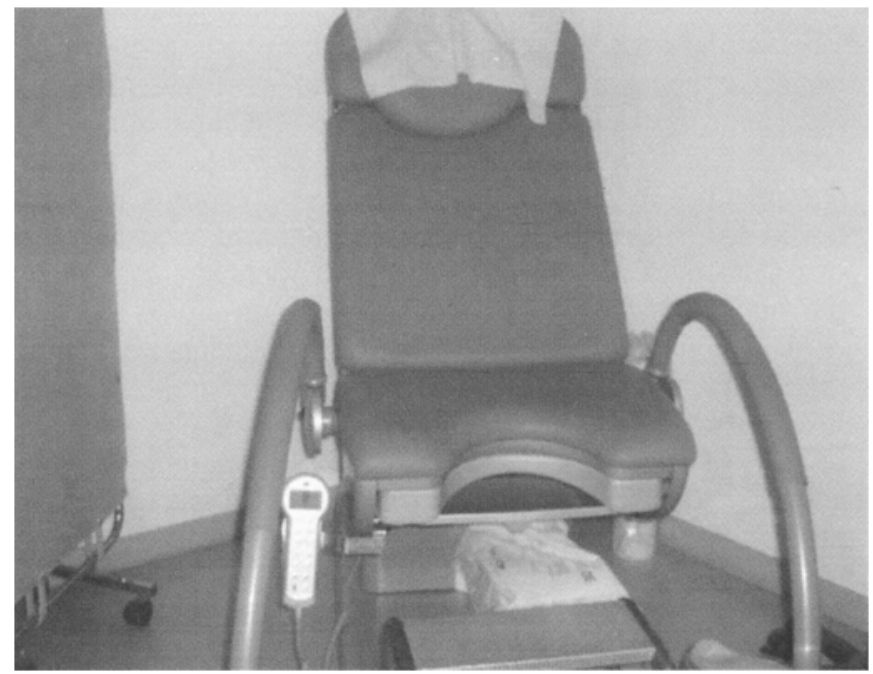

Figure 3 : Fauteuil d'examen adapté pour personne en situation de handicap. (Béatrice Idiard Chamois, Département mère-enfant IMM 2007)

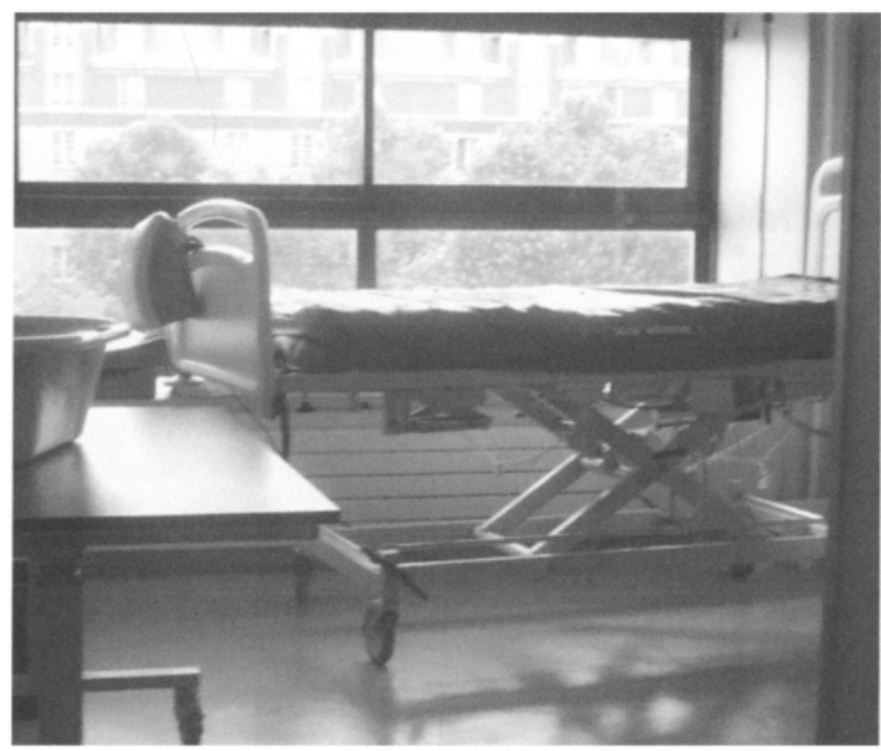

Figure 5 : Chambre avec lit et matelas anti escarres et table à langer réglable en hauteur manuellement. (Béatrice Idiard Chamois, Département mère-enfant IMM 2007)

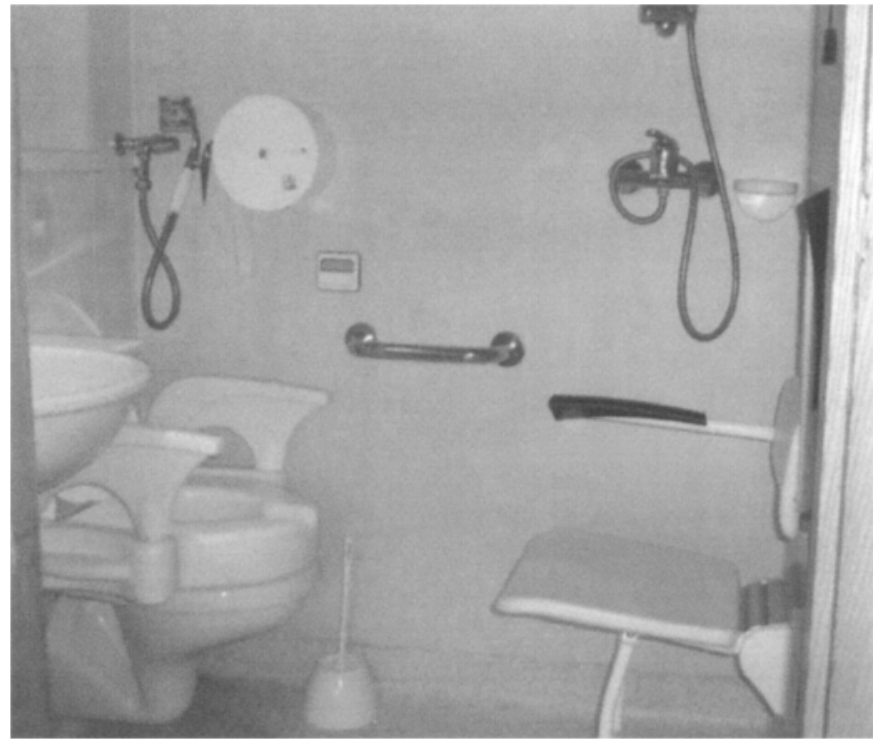

Figure 4 : Salle de bain et wc adaptés. (Béatrice Idiard Chamois, Département mère-enfant IMM 2007)

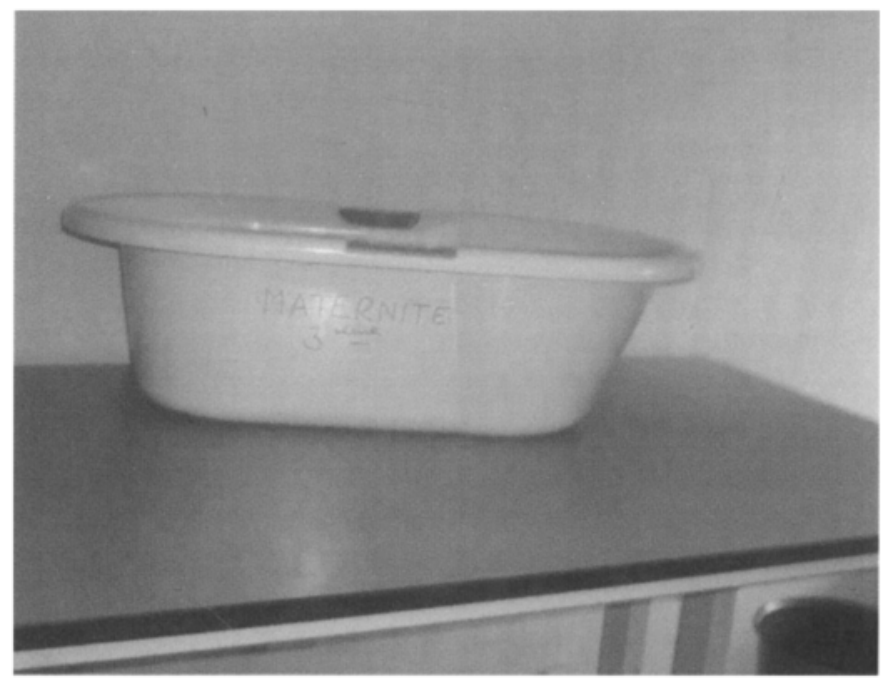

Figure 6 : Table réglable en hauteur manuellement et bajgnoire. (Béatrice Idiard Chamois, Département mèreenfant IMM 2007) 
situation de handicap, sauf peut-être pour une bonne cause comme celle de la recherche diffusée annuellement aux alentours de Noël ou bien le jour de l'Assomption où de nombreux fidèles tirent dans des petites charrettes des personnes malades, en situation de handicap (le vieux mythe de la charité chrétienne refait surface) : ambiguïté que celle de l'image de la personne insérée dans la société avec un travail et celle d'un enfant ou d'un adulte inspirant de la pitié, de la compassion liées à son état.

Je ne suis pas une professionnelle ou un anti partisan du diagnostic préimplantatoire, et je ne me permettrai pas de juger telle ou telle décision prise par un couple, et surtout aucune idéologie religieuse ne me hante, rassurez-vous, mais j'essaie de réfléchir un peu car atteinte d'une maladie génétique autosomique dominante considérée comme grave avec un pronostic vital maternel engagé, je me suis posée cette question : le DPI n'existant pas à l'époque, ai-je le droit de mettre un enfant au monde malgré le risque de transmission ? J'avais rencontré un vétérinaire en lieu et place du soutien nécessaire du corps médical dans l'hôpital où j'étais suivie. Je pense que ma décision n'a pas été prise au sérieux. Les médecins devaient probablement estimer que j'avais compris, que j'avais bien mesuré que je risquais d'avoir un enfant atteint, un enfant qui risquait de coûter cher à la sécurité sociale, bref que j'étais sagefemme et bien placée pour comprendre... La réponse m'a semblé évidente à ce moment là, oui, car aucune autre personne que moi-même peut décider à ma place de ce qui est " bon " ou " mal ", sauf mon propre enfant, qui me reprochera peut-être un jour d'avoir " osé " cette grossesse, " osé d'être mère " pour reprendre le titre du livre de Delphine Siegrist [15]. Dans une société qui ne fait aucune place au personnes en situation de handicap et où il faut légiférer afin d'avoir des droits, quand chaque jour est un combat non seulement avec sa maladie mais contre les NNCRH (normaux ne connaissant rien au handicap) et qu'il faut sans cesse prouver ce que l'on vaut, pas moins ni plus qu'une autre personne, et que renier sa maladie c'est aussi se renier soi-même, renier ce chemin parcouru avec des rencontres formidables, des gens qui m'ont tendu la main, d'autres mes ennemis que je remercie car il m'ont permis d'avancer, de franchir les obstacles.

Je suis convaincue que la difficulté de vivre une situation de handicap n'est pas liée à son degré, sa classification ou son origine mais comment on la vit, car on ne l'accepte jamais mais on vit avec ; un médecin qui recevait un malade présenté par un autre médecin et lui disait ceci « voilà ; il a été malade, il ne marche plus. Vous allez lui réapprendre à marcher "sa réponse a été " je ne vais pas lui apprendre à marcher, je vais lui apprendre à vivre sans marcher " [5].

Je pourrai encore écrire des centaines de pages sur les anecdotes tantôt prêtant à sourire tantôt le contraire mais il faut conclure, et je dirai que l'on peut faire autant de lois que l'on veut, nous changer de ministère (tantôt l'on dépend du ministère de la santé, tantôt celui du travail des solidarités et de la famille), est-ce que notre vie pour autant va s'en trouver chamboulée ? Va-t-on voir demain les entreprises embaucher leur quota d'handicapés (dans une société où le taux de chômage est important, beaucoup nous reprochent de prendre leur place), les écoles s'ouvrir à tous les enfants ? Les transports accessibles, l'information sur le handicap aux NNCRH (il y a du boulot !!!) ? Le matériel spécifique au handicap un peu moins cher ou mieux remboursé ? etc... On ne peut rien effacer et rien oublier de ce que les générations d'avant ont fait comme erreurs, ni celles que l'on créera encore. Notre rythme de vie est passé à la vitesse supérieure, tout doit aller vite, il n'y a pas de place pour les « trainards ", (le mot handicap fait parti de cette performance). Le vieillissement de la population française est en cours, des personnes saines deviennent à leur tour des personnes en situation de handicap grâce ou à cause des traitements, des technologies de plus en plus performantes. Pour celles-ci le gouvernement, la société a été incapable de prévoir et d'anticiper les conséquences naturelles d'une canicule. Nous profitons aussi de ces technologies. Alors que faire ? Je n'ai pas de solution miracle, moil'" ingérable », la " marginale » (adjectifs attribués par certains médecins), mais je pense que ce changement pourra venir des professionnels de santé, du secteur médico-social et de la volonté de chacun à ne pas regarder d'abord le handicap mais l'être humain avec ses défauts, ses qualités, bref tout ce qui fait son caractère... Je crois que si il y a un changement à faire, c'est au niveau de tous ceux qui travaillent de près ou de loin avec les personnes en situation de handicap c'est-à-dire, nous, vous. Je suis un citoyen lambda et à ma très petite échelle nous essayons modestement de changer les choses petit à petit, et si ce n'est qu'une goutte d'eau au milieu d'un océan, nous sommes fiers d'y avoir pensé et d'avoir agi.

Enfin je terminerai pas cette lecture paru dans le journal le Monde le 20/02/2007 écrit par un scénariste, Nicolas Journet atteint du même syndrome que moi, le syndrome de Marfan, à la suite du débat à l'assemblée en date du 07/02/2007 sur la révision de la loi de la bioéthique de 2004, qui, après avoir expliqué son parcours dit ceci :

" Génétiquement corrects : aujourd'hui on parle de rendre le diagnostic prénatal systématique pour cette maladie, pour ma maladie. Bonne idée. Les enfants atteints peuvent être suivis dès leur naissance. Mais encore faut-ils qu'ils naissent. Qui expliquera aux parents que maladie génétique et bonheur ne sont pas des termes antinomiques ? Les médecins ? Par expérience j'en doute. Et pourtant je suis très heureux. Bien plus heureux que beaucoup de génétiquement corrects. Et qu'en sera-t-il du diagnostic préimplantatoire ? La précédente loi bioéthique d'Août 2004 en restreint la pratique aux maladies « gravement invalidantes, à révélation tardive, et mettant prématurément en jeu le diagnostic vital ", Marfan en fera-t-il partie ?

Est-ce à dire que les vies de Lincoln, Paganini, Rachmaninov, Marie Stuart, Peter Crouch, Ronny Turiaf, et tant d'autres anonymes aussi alternativement superbes et médiocres que n'importe qui sur terre, ont été ou sont inutiles ? Notre société ne veut plus affronter la mort, ne veut plus du hasard. Elle croit pouvoir contrôler son destin. Quitte à sombrer dans l'eugénisme, quitte à renouer avec le nazisme. Quelle illusion ! Comme l'écrivait Jean Rostand : " Quand l'habitude sera prise d'éliminer les monstres, de moindres tares feront figure de monstruosités. De la suppression de l'horrible à celle de l'indésirable, il n'y a qu'un pas... Cette société nettoyée, assainie, cette société sans déchets sans bavures, où les normaux et les forts bénéficieraient de toutes les ressources qu'absorbent jusqu'ici les anormaux et les faibles, cette société renouerait avec Sparte et ravirait les disciples de Nietzsche, je ne suis pas sûr qu'elle mériterait encore d'être appelée une société humaine ".

L'Allemagne a retenu les leçons de son histoire et interdit le diagnostic préimplantatoire. La France doit faire de même sous peine de rentrer en contradiction avec ses propres lois. Article L214-1 du code pénal : " le fait de mettre en œuvre une pratique eugénique tendant à l'organisation de la sélection des personnes 
est puni de trente ans de réclusion criminelle et de 7500000 euros d'amende ". Et certains généticiens doivent apprendre l'humilité. II y a une limite au progrès scientifique. Cela s'appelle l'éthique. Cela s'appelle l'intelligence.

Quant à moi... Jusqu'à la semaine dernière, j'avais un doute, mais désormais mon choix est arrêté. Je ferai des enfants. Pour transmettre le « mal ». Pour combattre la connerie par l'amour.

\section{RÉFÉRENCES}

1. Conférence de presse du 9 février 2006 de Philippe Bas, ancien ministre délégué à la sécurité sociale, aux personnes âgées, aux personnes handicapées et à la famille.

2. DEBUREAUX M.: Hier aujourd'hui à deuX mains. Langue des Signes, Edition Publication, 2004.

3. DELAPORTE Y. : directeur de recherche au CNRS, attaché au laboratoire d'anthropologie urbaine, se consacre depuis 1994 à l'exploration du monde sourd. Les sourds c'est comme ça. Editions de la maison des sciences de l'Homme, Paris, 2002.

4. MOODY B., VOURC'HA., GIROD M., HOF D. : La langue des Signes Histoire et grammaire. Editions IVT, 1988.

5. L'annonce anténatale et postnatale du handicap. Mission handicap Espace Ethique. les dossiers de l'AP/HP, 2001

6. STAHLA., TOURAME P. : Le handicap dans l'Histoire. Cahiers du SYNGOF, 2006, $59: 40-47 ; 60: 35-39$.

7. LOI $n^{\circ}$ 57-1223 du 23 novembre 1957 sur le reclassement professionnel des travailleurs handicapés.

8. LOI $n^{\circ} 75-534$ du 30 juin 1975 d'orientation en faveur des personnes handicapées (mise à jour du 24 janvier 1997).

9. LOI $n^{\circ} 87-517$ du 10 Juillet 1987 en faveur de l'emploi des travailleurs handicapés (J.O. du 12 juillet 1987).

10. LOI $n^{\circ} 90-602$ du 12 juillet 1990 relative à la protection des personnes contre la discrimination en raison de leur état de santé ou de leur handicap (J.O. du 13 juillet 1990).

11. LOI $n^{\circ}$ 91-663 du 13 juillet 1991 portant diverses mesures destinées à favoriser l'accessibilité aux personnes handicapées des locaux d'habitation, des lieux de travail et des installations recevant du public (J.O. du 19 juillet 1991).

12. LOI $n^{\circ} 2002-73$ du 17 janvier 2002 rénovant l'action sociale et médicosociale.

13. LOI $n^{\circ} 2004-1486$ du 30 décembre 2004 portant création de la haute autorité de lutte contre les discriminations et pour l'égalité.

14. LOI 2005-102, 2005-02-11 du 11/02/2005 sur l'égalité des droits et des chances, la participation et la citoyenneté des personnes handicapées.

15. SIEGRIST D. : Oser être mère, maternité et handicap moteur. Edition DOINS, 2003.

16. Rapport de l'enquête HID faite par l'INSEE : Handicaps-incapacités dépendance (premiers travaux d'exploitation de l'enquête HID, colloque scientifique, Montpellier, 30 novembre - 1 décembre 2000 , coordination Christel Colin et Roselyne Kerjosse) 16, juillet 2001.

17. Saga Sciences, dossiers scientifiques, 'science et handicap' (la science au service des handicapés), CNRS, 2003.

18. Site Officiel du Musée National de la Sécurité Sociale, histoire des origines de la sécurité sociale : musee-assurance-maladie.com

19. Site du Syndicat des Ophtalmologistes de France : www.avh.asso.fr

20. Site de l'Association Valentin Haüy : www.snof.org

21. STIKER H.J. : Corps infirmes et sociétés. Paris, Aubier Montaigne, 1982.

Manuscrit reçu : septembre 2007 ; accepté septembre 2007.

Communication FFER, octobre 2007, Amiens.
Remerciements :

Un grand merci aussi aux professeurs sourds pour l'enseignement de la LSF et de Thierry Ruchot Chercheur historien sourd pour l'histoire.

\section{ABSTRACT}

\author{
The new disability law
}

\section{Béatrice IDIARD CHAMOIS}

Although, over the centuries, disabled persons have been successively considered to be an image of evil, pity, or sin, what image and what rights are provided by the French law of 11/02/2005 on equal rights, participation and citizenship of disabled persons? Isn't there still an enormous gap between written laws and what really happens in everyday life, as dozens of laws have been added since the first law voted in the 19th century? The vocabulary has also changed over the years from mutilated, to handicapped, to disabled.

The marked differences between official figures and figures derived from the HID (Handicap Independence Incapacity) survey conducted between 1998 and 2001, clearly illustrates the difficulty of defining disability. With ageing of the French population aging and improvement of technologies, the number of disable persons will increase considerably over the years to come. Does the new law of 11/02/2005 provide a solution to this urgent situation? Has it identified all of the problems concerning accessibility, human assistance, schooling, rights of the disabled or is it just another law on disability, which is more useful to the legislator than to the people concerned?

The new law focuses on four points. First of all, the right to compensation by creating a disability pension, which replaces third party benefits and professional fees benefits and adult disability pension, the right to schooling for all disabled children, the right to employment, and finally the creation of departmental disability centres, staffed by the various actors currently involved in the care of the disabled (Conseil Général, DDASS, CPAM, CAF, etc...).

Creation of the disability pension is now based on five main types of assistance : human assistance, technical assistance, adaptation of the home and the vehicle as well as excess transport costs, animal assistance, and finally exceptional and specific aids.

Apart from direct assistance to disabled persons, the new law is also designed to improve accessibility (law and decrees already in application since 1991 and 1994) to transport, public places, etc. However, the law also gives another definition of accessibility by extending its scope to all aspects of disability (motor, sensory, cognitive, mental, etc.). This accessibility must be implemented within defined deadlines: 3 years for transport, 5 years for universities and prefectures, and 10 years for other public buildings. 
Another innovation of this law is that of the initial training and continuing education of healthcare professionals of the medical and social sector (cause of disability, therapeutic innovations, educational innovations, reception and supportive care of disabled persons, announcement of disability, etc.).

However, as so little progress has been made the over the centuries, many disabled people will be left by the wayside. Although politicians have now become aware of the problem, the errors, delays, and imperfections of this new law will allow tolerance rather than insertion of the disabled in the years to come.

We must develop a different vision of disability, as illustrated by the creation, in 2002, of a European Commission network called "Design for All" to develop systems that can be used by healthy as well as disabled persons.

Disabled persons are also trying to find solutions concerning parenthood and childbirth, as although the law has defined "all" aspects for healthy parents with a disabled child, no measures have been taken for disabled parents. The MotherChild Department of the Institut Mutualiste Montsouris is developing a programme for these future parents with the help of ESCAVIE (Espace Conseil pour l'autonomie en milieu ordinaire de vie) in collaboration with the occupational therapist and social worker. A disabled parents association has also been formed in the Mother-Child Department.

A last point does not directly concern the new law, but the bioethics law of August 2004 and preimplantation diagnosis, which was the subject of a recent debate concerning its revision. The old myth of birth control has resurfaced. Unfortunately politicians collude with the media to present this stereotyped image of the perfect, beautiful, normal baby.

Key words: disability, disability benefits, training, accessibility, parentality, bioethics law 